\title{
Conocimientos, actitudes y prácticas de bioseguridad de los estu- diantes de las carreras del área de la salud de la UNAH, realizado en la ciudad universitaria en 2013
}

Ada Argentina Zelaya Discua ${ }^{1}$

\section{RESUMEN}

El presente estudio identifica los riesgos biológicos, químicos y físicos a los que están expuestos los estudiantes de las carreras del área de la salud, así como el conocimiento que tienen los estudiantes de estos riesgos y las prácticas de bioseguridad que se llevan a cabo durante el proceso enseñanza-aprendizaje. También relaciona los conocimientos y la percepción de riesgo con las prácticas de prevención que llevan a cabo los estudiantes de las diferentes carreras. Se utilizó un cuestionario sobre conocimiento, medidas de prevención, percepción de riesgo, controles administrativos y controles de ingeniería. El $83 \%$ de los estudiantes identifica el riesgo biológico y el $5 \%$ el riesgo químico. El $58 \%$ conoce la forma de eliminar desechos bioinfecciosos, el $70 \%$ identifica el VIH como un virus que se transmite por fluidos corporales. Solamente el $37 \%$ utiliza bolsas rojas para eliminar los desechos. El 89 $\%$ de los estudiantes consideran que el personal de salud está más expuesto a riesgos laborales que en otro tipo de carreras. Existe una adecuada percepción de riesgo de los estudiantes, el $84 \%$ se considera en riesgo de adquirir una enfermedad durante el proceso enseñanza-aprendizaje. Se encontró relación directa entre el conocimiento que tienen los estudiantes de los riesgos a los que se exponen en su carrera y las prácticas de prevención que realizan. No se encontró relación entre percepción de riesgo de contraer una infección durante el proceso-enseñanza aprendizaje y la puesta en práctica de medidas de prevención.

Palabras claves: bioseguridad, riesgo, percepción de riesgo, prevención, equipo de protección personal.

\footnotetext{
${ }^{1}$ Beneficiaria de una beca sustantiva de la DICYP. Profesora investigadora de la Escuela de Microbiología, Facultad de Ciencias, UNAH: adazelaya@yahoo.com
} 


\section{ABSTRACT}

This study identifies the biological, chemical and physical risks to which students from health related careers are exposed to, as well as the knowledge students have of these risks and the practice of bio-safety during the teaching-learning process. It also relates knowledge to the risk perception used by students from different career in preventive practices. A questionnaire was used to gather the information about preventive measures, risk perception, administrative controls and engineering controls.

About $83 \%$ of the students are able to identify biological risks and $5 \%$ identify the chemical risks. Approximately $58 \%$ of the students know how to get rid of biochemical waste, and $70 \%$ identify the HIV as a virus that is transmitted by bodily fluids. Only $37 \%$ of the students use red bags to get rid of the waste. Nearly $89 \%$ of the students consider that health professionals are more exposed to biological risks than other career professionals. There is an adequate risk perception, of $84 \%$ who consider themselves in risk of contracting a disease in the teaching-learning process. There is a direct relationship between what students know about the risks they are exposed to in their career and the preventive practices they perform. There was no relationship found between the risk perception of getting infected during the teaching-learning process and the implementation of preventive measures.

Keywords: biosafety, risk, risk perception, prevention, personal protection equipment. 


\section{INTRODUCCIÓN}

Los trabajadores del área de la salud deben poseer competencias que los habiliten para realizar su trabajo de tal modo que mitiguen los riesgos de infectarse o dañarse con variados agentes biológicos, químicos y físicos a los que están expuestos en su trabajo diario. Asimismo, deben tomar todas las medidas necesarias y adecuadas para evitar que alguno de estos agentes llegue al ambiente y la comunidad.

Estas competencias se desarrollan mediante un entrenamiento planificado, el cual implica no solo conocimientos teóricos, sino prácticas en ambientes adecuadamente diseñados y equipados para lograr tal fin (Ferreira, Barrozo y García, 2004). Al respecto, la presente investigación identifica los conocimientos sobre bioseguridad que han incorporado los estudiantes de las carreras del área de la salud de la UNAH en su formación académica, la actitud que manifiestan ante los riesgos biológicos, químicos y físicos propios de su quehacer y las prácticas de protección que se observan durante el proceso enseñanza-aprendizaje en los laboratorios y clínicas de docencia. Los resultados obtenidos serán utilizados en el Centro Nacional de Capacitación en Bioseguridad (CENCAB) para elaborar los programas y contenidos de las capacitaciones que se desarrollarán con docentes, alumnos y personal auxiliar de las diferentes carreras del área de la salud.

La falta de estudios de bioseguridad que determinen el nivel de conocimiento sobre las normas universales de bioseguridad entre estudiantes de las carreras del área de la salud de la UNAH y la correlación de este con la percepción de riesgo y las medidas de contención, así como el elevado riesgo de infecciones transmisibles de gran impacto en la comunidad como el VIH/sida o el virus de la hepatitis B, llevó a plantear este trabajo de investigación a fin de contar con la información necesaria (línea base) para diseñar el programa de capacitación en cada carrera del área de la salud y establecer mecanismos que permitan mejorar la percepción de riesgo y el comportamiento seguro ante el riesgo biológico, químico o físico al que diariamente se exponen los estudiantes, docentes y personal auxiliar en las carreras del área de la salud de la UNAH.

El estudio analizó cada una de las carreras individualmente, en esta oportunidad se presenta únicamente el consolidado de todas las carreras del área de la salud, pero existe un informe individual por carrera, los que ya se han entregado a las diferentes carreras y están siendo utilizados en el CENCAB. 


\section{MÉTODO}

El presente estudio es de tipo descriptivo y transversal, que evalúa conocimientos, actitudes y prácticas de bioseguridad de los estudiantes de las carreras del área de la salud de la UNAH. Analiza si existe relación entre el conocimiento del riesgo, de las medidas de prevención, la percepción de riesgo, las condiciones de infraestructura con la práctica de las medidas de bioseguridad en cada una de las carreras. El estudio se llevó a cabo durante el año 2013.

Se define al alumno que participa en la investigación, como el estudiante que cursa las asignaturas de Microbiología para Medicina (MB-610), Microbiología para Odontología (MB-111), Microbiología para Enfermería (MB-110), Bioquímica Clínica I (MB-159); Bacteriología Médica (MB-066) de la Carrera de Microbiología y Bioquímica (QQ-321) de la Carrera de Química y Farmacia; asimismo, estudiantes de segundo y tercer año de las Carreras de Radiotecnología y Terapia Funcional.

El tamaño de la muestra fue de 1,055 estudiantes. Las asignaturas fueron seleccionadas por conveniencia, con el requisito de que fueran asignaturas que el estudiante cursa, al menos, en el tercer año de la carrera, exceptuando las carreras de Terapia Funcional y Radiotecnología, que incluyen alumnos de segundo y tercer año.

A todos los alumnos de las asignaturas escogidas se les aplicó el cuestionario de 80 preguntas. Una vez recolectada la información se elaboró la base de datos en EPI INFO 7, se determinó la frecuencia y porcentaje para las diferentes variables y se realizó una prueba no paramétrica de Chi cuadrada (prueba estadística para evaluar hipótesis acerca de la relación entre dos variables categóricas); se utilizó para la variable dependiente: cumplimiento de normas y para las variables independientes: conocimiento de normas de bioseguridad y percepción de riesgo. Los resultados se organizaron en gráficos y tablas.

\section{RESULTADOS}

La muestra fue de 1,055 estudiantes, 741 (70.3\%) del sexo femenino y $313(29.7 \%)$ del sexo masculino. El $97 \%$ tenían edades en el rango de 18 a 29 años, $86 \%$ de los estudiantes cursaban el 3 año de su respectiva carrera y el $14 \%$ el segundo año (ver tabla 1). 
Tabla 1. Número y porcentaje de participación de los estudiantes de las diferentes carreras del área de la salud

\begin{tabular}{llcc}
\hline Carrera & Asignatura & $\begin{array}{c}\text { Número de } \\
\text { estudiantes }\end{array}$ & $\%$ \\
\hline Enfermería & Microbiología MB-110 & 105 & 10.0 \\
\hline Química y Farmacia & Bioquímica QQ-321 & 181 & 17.2 \\
\hline Medicina & Microbiología MB-610 & 396 & 37.5 \\
\hline Microbiología & Bioquímica Clínica MB 159 & 108 & 10.0 \\
\hline Odontología & Microbiología MB-111 & 168 & 16.0 \\
\hline Radiotecnología & Segundo y tercer año & 25 & 2.5 \\
\hline Terapia Funcional & Segundo y tercer año & 72 & 6.8 \\
\hline Total & & 1,055 & 100.0 \\
\hline
\end{tabular}

\section{Tipos de riesgo}

Los estudiantes de las carreras del área de la salud están expuestos a riesgo biológico, químico y físico. El tipo de riesgo a los que se exponen los estudiantes depende de la naturaleza de la carrera, la exposición y del tipo de trabajo que se realice. En el gráfico 1 se observa el riesgo biológico que es identificado por el mayor número de estudiantes. 
Gráfico 1. Tipos de riesgo identificados por los estudiantes de las carreras del área de la salud de la UNAH

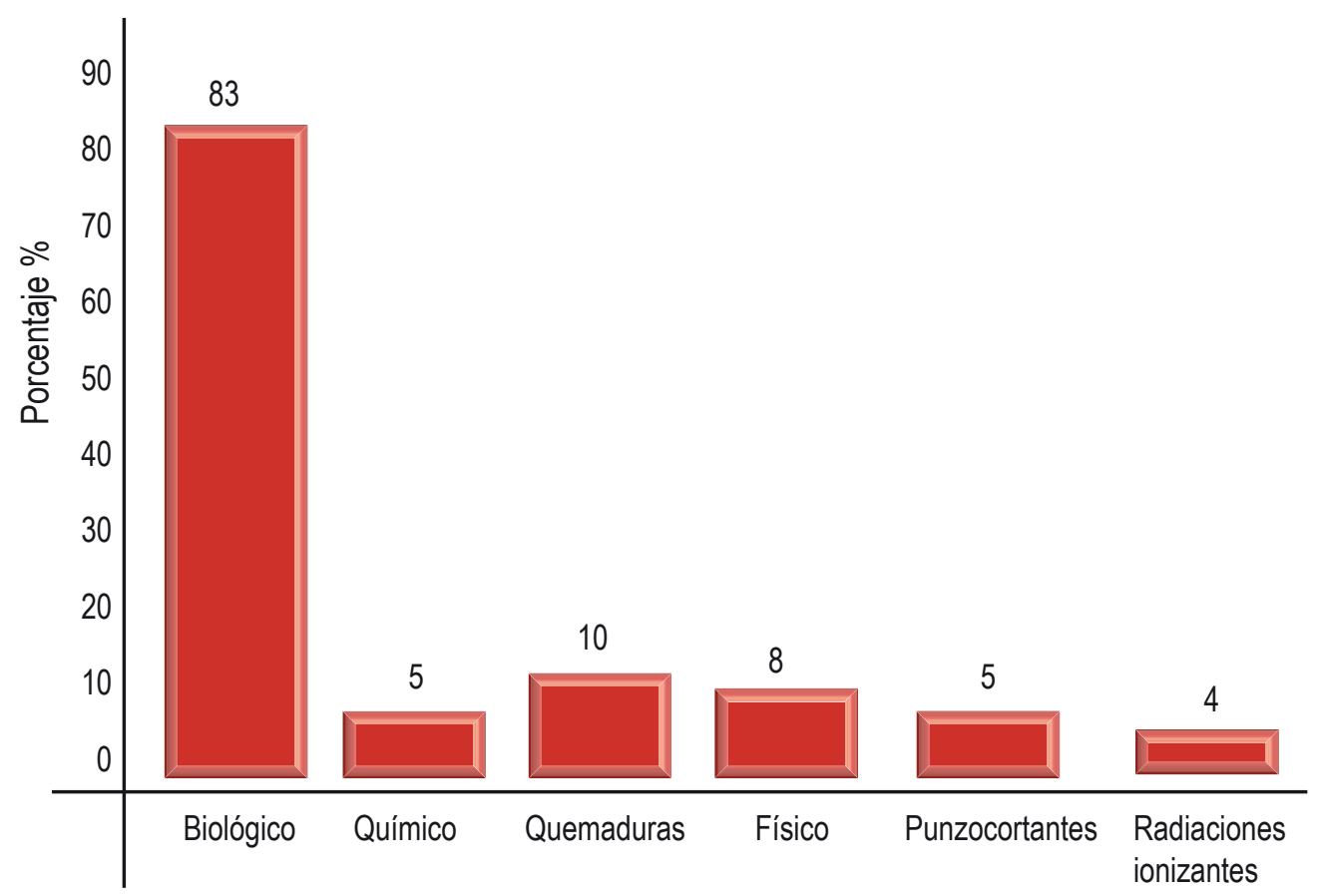

2. Conocimiento de las medidas de prevención

El conocimiento que tienen los estudiantes con respecto a las infecciones transmitidas por sangre y otros fluidos corporales, sobre los desinfectantes y la disposición de desechos se observa en la tabla 2.

Las hojas de seguridad de las sustancias químicas (MSDS) contienen la información necesaria para el uso, manejo y medidas de seguridad, de modo que es necesario que los usuarios de estos reactivos conozcan la información contenida en estas hojas de seguridad. Pero, se observa diferente nivel de conocimiento al respecto, así, $84 \%$ de los estudiantes de la Carrera de Química y Farmacia conocen las hojas MSDS, 56 $\%$ de Radiotecnología, $46 \%$ de Microbiología, $38 \%$ de Odontología y $26 \%$ de Enfermería. Los alumnos de Medicina y de la Carrera de Terapia Funcional no mencionan el conocimiento de las hojas de seguridad. 
Tabla 2. Conocimientos relacionados con la bioseguridad que tienen los estudiantes de las carreras del área de la salud

Conocimientos generales de bioseguridad

\section{Número de}

estudiantes medios de transmisión del virus de la inmunodeficiencia humana (VIH)

Identifican la sangre como uno de los medios de transmisión del virus de la hepatitis B

Identificaron el virus de la hepatitis $B$ como el que permanece durante más tiempo viable fuera del organismo

Conocen la concentración de cloro adecuada para ser utilizado como 206 desinfectante

Conocen la concentración de alcohol adecuada para ser utilizado como 599 desinfectante

Conocen la concentración del cloro que se compra en el comercio (supermercados y pulperías)

Conocen la forma adecuada de eliminar los desechos bioinfecciosos provenientes de clínicas y laboratorios (bolsas rojas) 
La amenaza de una pinchadura con un objeto punzocortante que existe en la práctica de las carreras relacionadas con la salud, requiere del conocimiento de las medidas de prevención y mitigación del daño en caso de que este ocurra. El gráfico 2 muestra las medidas de mitigación que aplicarían los estudiantes en caso de sufrir una pinchadura.

Gráfico 2. Medidas de mitigación identificadas por los estudiante en caso de sufrir una pinchadura con un objeto punzocortante para evitar la transmisión de enfermedades

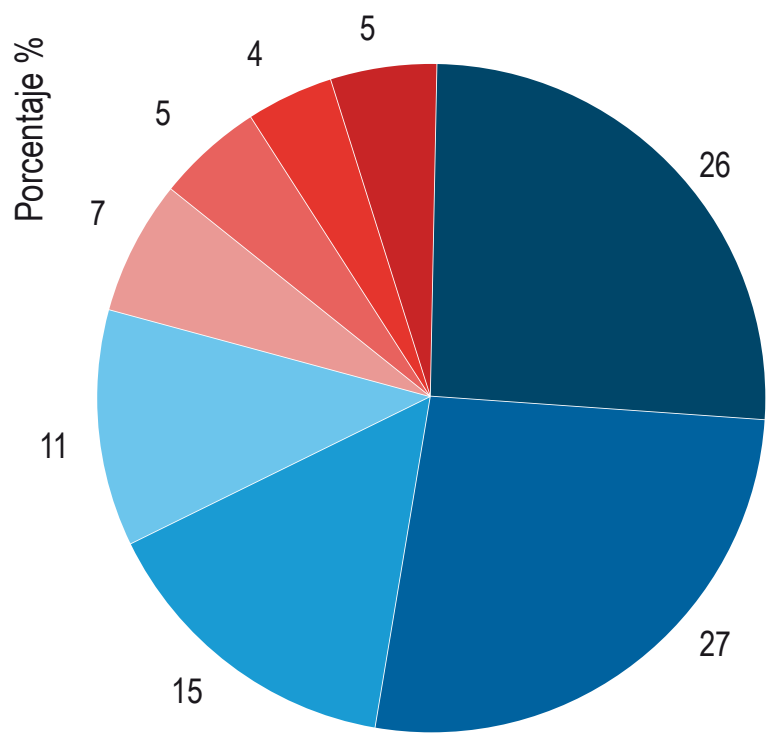

Ir al médico inmediatamente Lavar con desinfectante Lavar con agua Tomar antirretrovirales No contesta Seguir protocolo Comunicar al profesor No contestó

Los aerosoles y salpicaduras son una fuente importante de transmisión de microorganismos y de contaminación química, el conocimiento que tienen los estudiantes de las medidas de bioseguridad para evitar la transmisión de enfermedades por este medio se aprecia en el gráfico 3.

El equipo de protección personal que el alumno de las diferentes carreras considera que se debe utilizar para evitar la transmisión de infecciones por sangre u otros fluidos corporales en las diferentes carreras se observa en el gráfico 4. 
Gráfico 3. Medidas de bioseguridad contra los aerosoles identificadas por los estudiantes del área de la salud

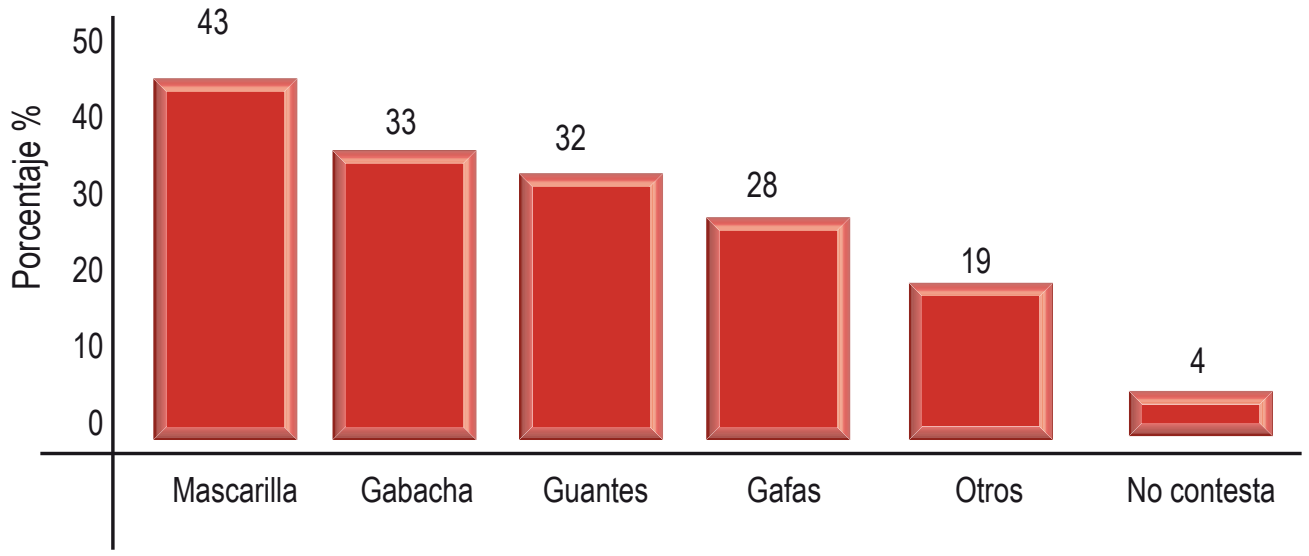

Gráfico 4. Equipo de protección personal identificado por los estudiantes para evitar la transmisión de enfermedades por contacto con sangre u otros fluidos corporales

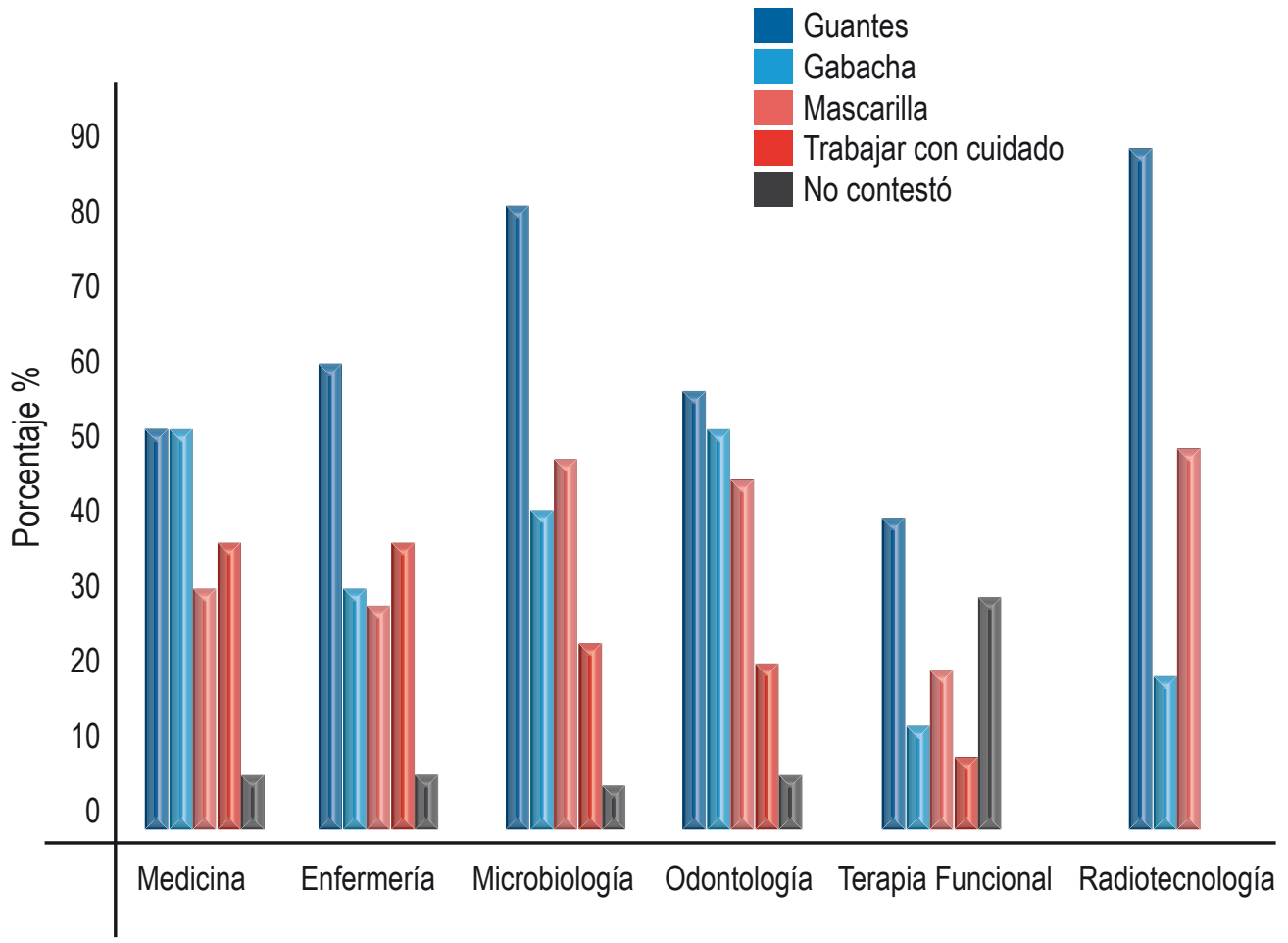




\section{Percepción de riesgo}

Ante la afirmación: "El personal del área de la salud está expuesto a más riesgos laborales que otro tipo de profesional", los resultados que arrojaron los estudiantes se describen en el gráfico 5.

Gráfico 5. Percepción de riesgos laborales en los estudiantes, en porcentaje

Muy en desacuerdo

En desacuerdo

Ni de acuerdo ni desacuerdo

De acuerdo

Muy de acuerdo

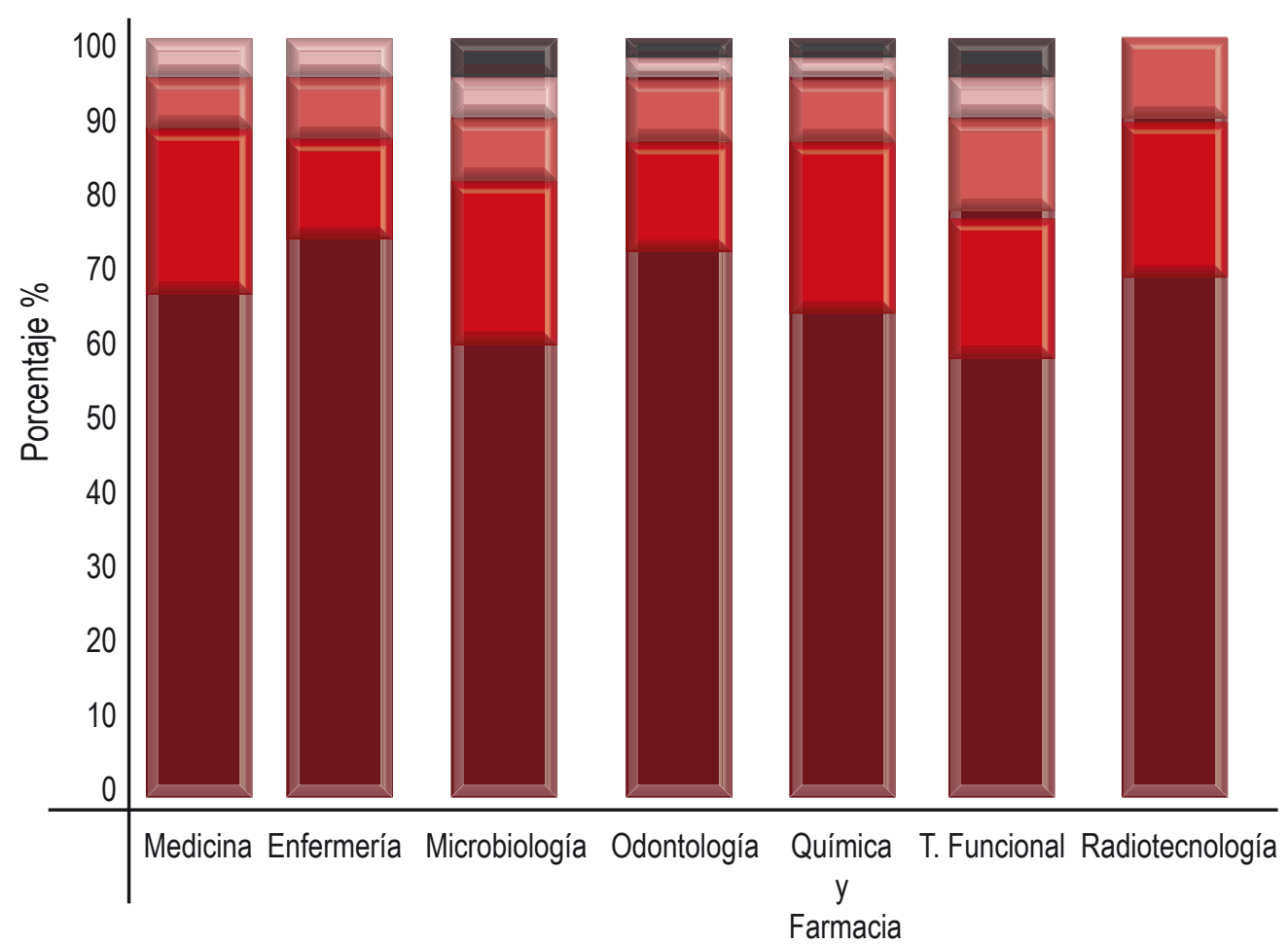

El $84 \%$ (886 estudiantes) se considera en riesgo de adquirir una enfermedad durante el proceso enseñanza- aprendizaje. Las razones por las que se consideran en riesgo se describen en la tabla 3. 
Tabla 3. Riesgo de adquirir una infección durante el proceso enseñanza-aprendizaje en los estudiantes

\begin{tabular}{|lcc|}
$\begin{array}{l}\text { Razones por las que se consideran en riesgo los } \\
\text { estudiantes de las carreras del área de la salud }\end{array}$ & $\begin{array}{c}\text { Número de } \\
\text { estudiantes }\end{array}$ & $\%$ \\
\hline $\begin{array}{l}\text { Puede ocurrir un accidente } \\
\text { Tenemos compañeros descuidados }\end{array}$ & 570 & 54 \\
\hline Hay muchos estudiantes en poco espacio & 556 & 53 \\
\hline $\begin{array}{l}\text { Las instalaciones o el equipo no son adecuados } \\
\text { Mal uso de materiales y equipo }\end{array}$ & 471 & 45 \\
\hline $\begin{array}{l}\text { Poco control y vigilancia de los maestros } \\
\text { Muestras clínicas muy contaminadas }\end{array}$ & 348 & 33 \\
\hline $\begin{array}{l}\text { No exigen el cumplimiento de medidas de } \\
\text { bioseguridad }\end{array}$ & 1277 & 14 \\
\hline No cumplo las medidas de bioseguridad & 125 & 12 \\
\hline No conozco las formas de protegerme & 122 & 7 \\
\hline Otras & 69 & 12 \\
\hline
\end{tabular}

Del total de estudiantes, el $47 \%$ (487 alumnos) reporta utilizar las medidas de bioseguridad siempre. En el gráfico 6 se observa el grado de cumplimiento de las medidas de prevención en las diferentes carreras. 
Gráfico 6. Frecuencia del cumplimiento de las medidas de bioseguridad de los estudiantes de las carreras del área de la salud

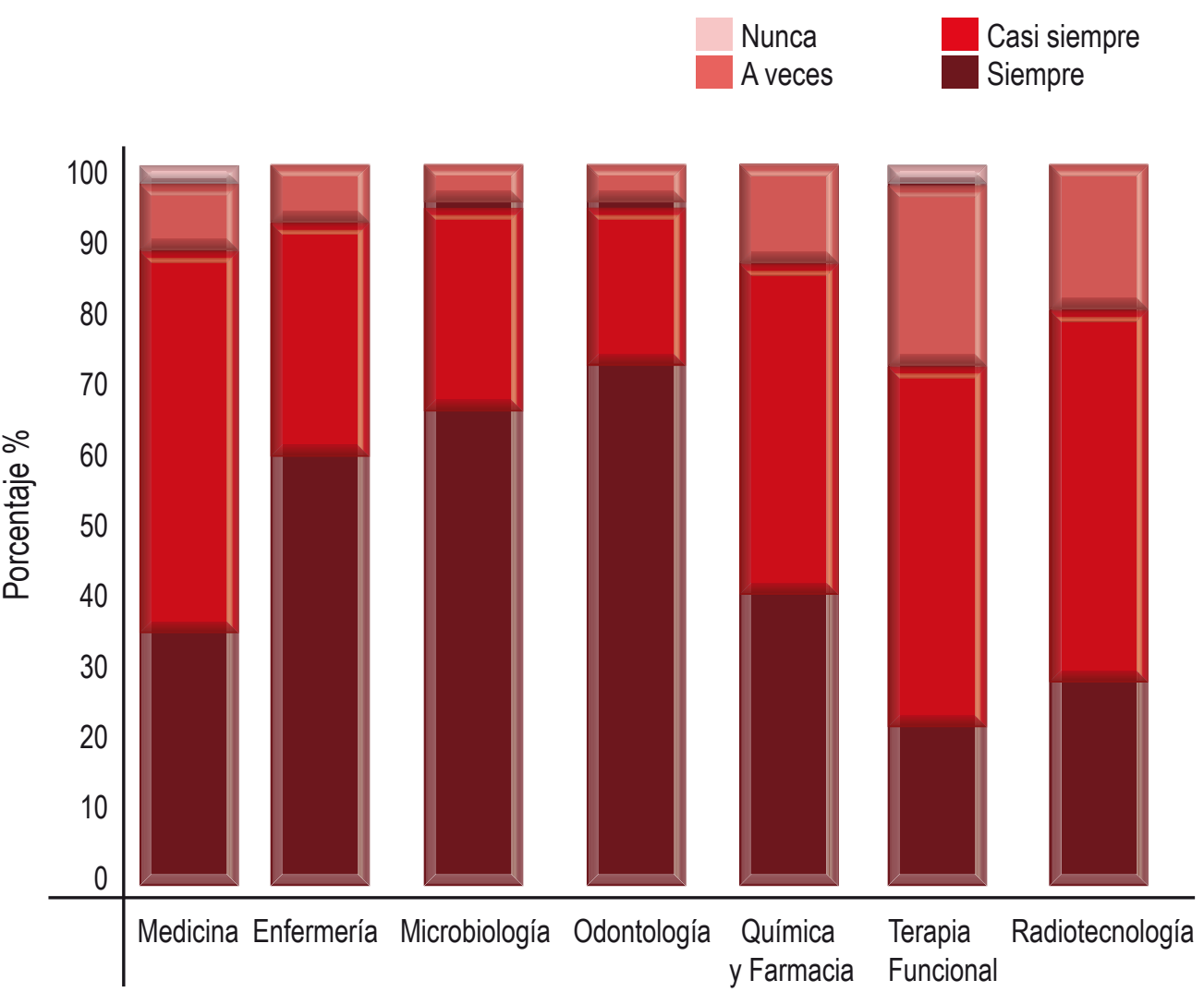

El $84 \%$ (886) de los estudiantes se considera en riesgo de adquirir una infección durante sus clases prácticas. En el gráfico 7 se muestra que los estudiantes se consideran en riesgo y los que cumplen con las medidas de bioseguridad en las diferentes carreras.

Las razones por las cuales los estudiantes aplican las medidas de bioseguridad son principalmente por el deseo de protegerse ante los riesgos existentes $(87 \%)$, porque han adquirido los conocimientos necesarios para llevar a cabo prácticas de protección (53\%), porque lo exigen las normas (37\%) y porque lo piden los profesores (36\%). En el gráfico 8 se observa el grado de protección que perciben los estudiantes en cada una de las carreras. 
Gráfico 7. Relación entre percepción de riesgo y el cumplimiento de medidas de bioseguridad

- Se considera en riesgo de adquirir una infección durante el proceso enseñanza apredizaje
Cumple con las medidas de bioseguridad siempre

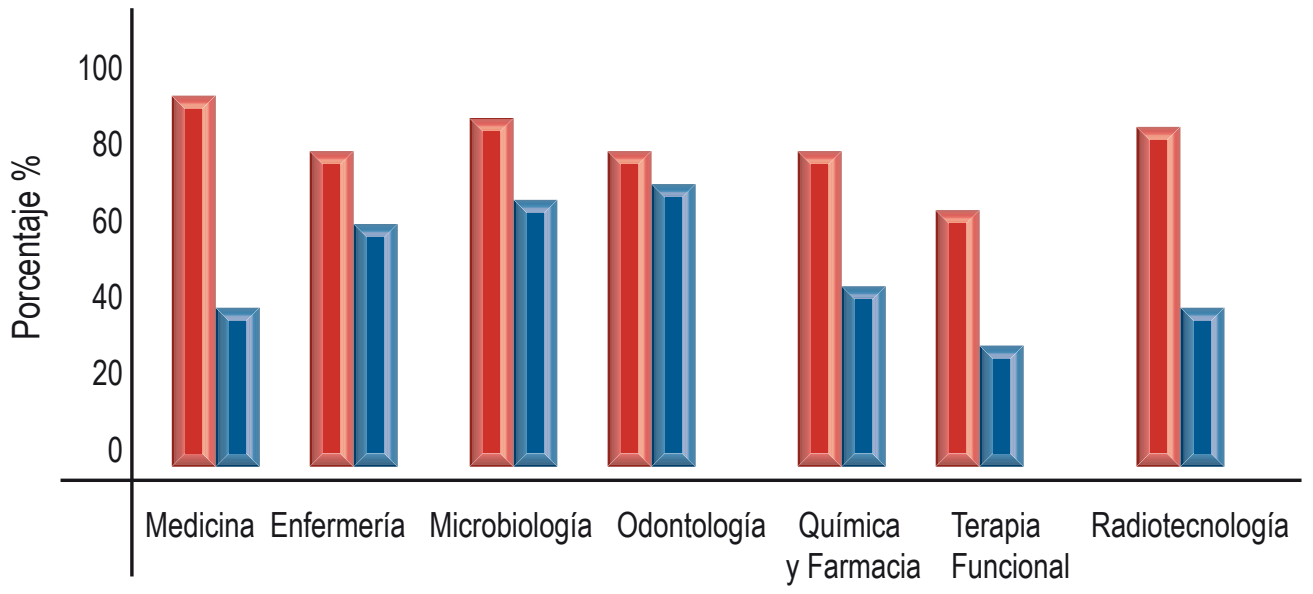

Gráfico 8. Grado de protección que perciben los estudiantes en cada una de las carreras del área de la salud de la UNAH

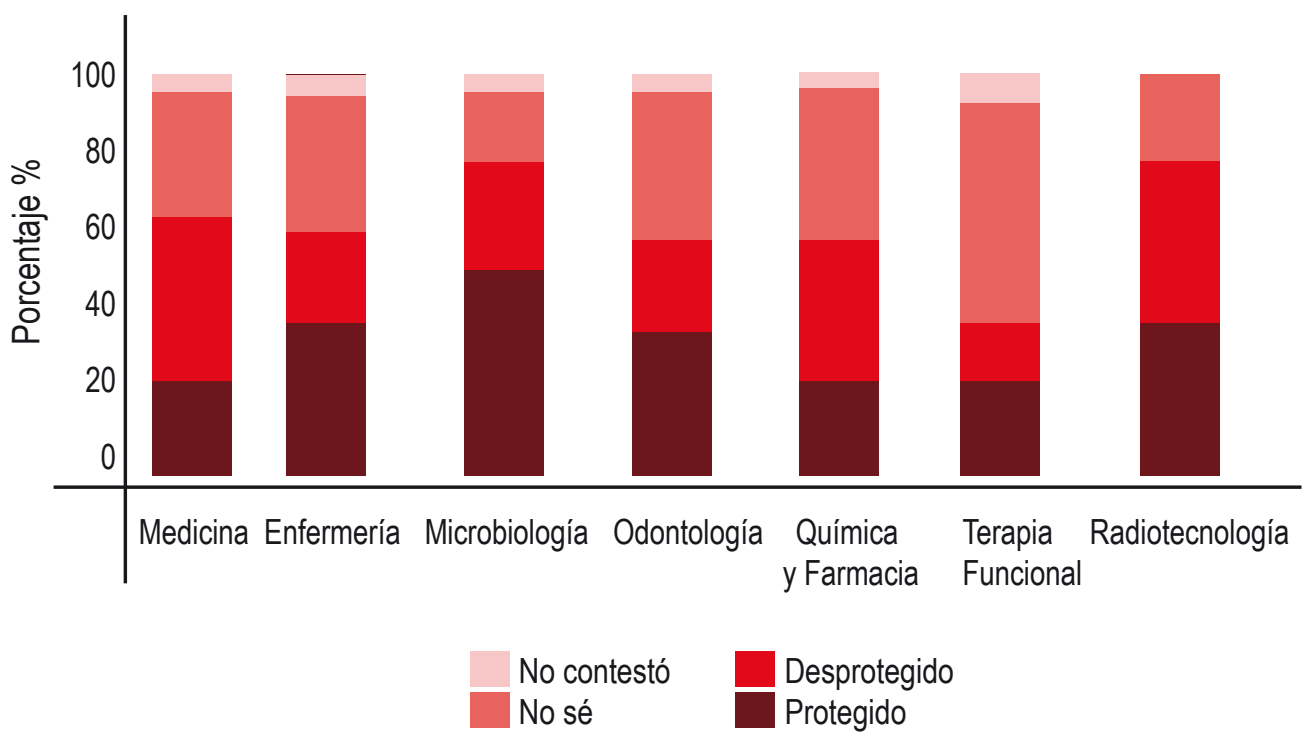




\section{Prácticas de bioseguridad}

El $61 \%$ de los estudiantes (642) reportan el uso de equipo de protección personal (EPP): gabacha, guantes, mascarilla, durante sus prácticas. El promedio de guantes utilizados diariamente por los estudiantes varía desde un par (24\%), dos pares (21\%), tres pares (12\%) y los que no utilizan guantes (19\%).

El material bioinfeccioso o contaminado que sale de laboratorios, clínicas, hospitales, clínicas odontológicas, etc., debe ser depositado y esterilizado antes de salir del lugar en donde se generó para ser eliminado. En el gráfico 9 se observan los diferentes métodos que utilizan los estudiantes para eliminar los desechos. Otras prácticas de bioseguridad que realizan los estudiantes se observa en la tabla 4.

Gráfico 9. Recipientes utilizados por los estudiantes de las carreras del área de la salud para eliminar el material bioinfeccioso durante sus prácticas

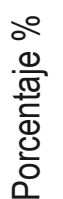

22

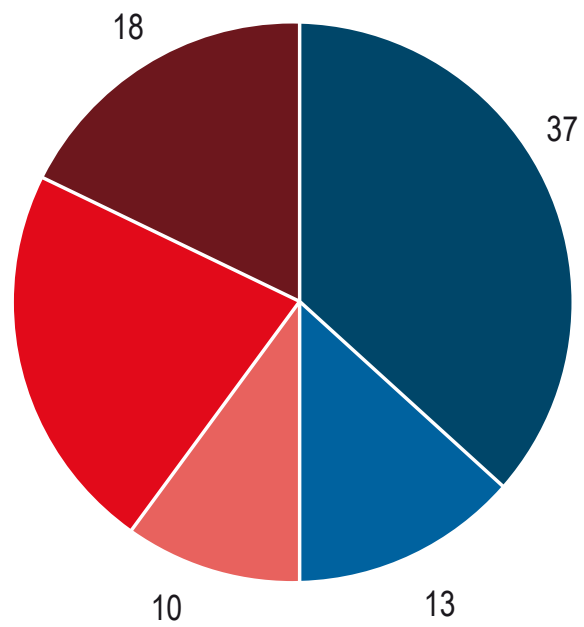

El $11 \%$ (120) de los estudiantes reportan haber sufrido pinchadura con objeto punzocortante durante sus actividades académicas; el $55 \%$ manifestó que ha sufrido pinchadura y que reportó el incidente al profesor.

Asimismo, los estudiantes mencionan haber adquirido conocimientos sobre bioseguridad en las diferentes carreras. En el gráfico 10 se observa el porcentaje de estudiantes por carrera. 
Tabla 4. Prácticas de bioseguridad que realizan los estudiantes de las carreras del área de la salud

\begin{tabular}{lcc} 
Práctica de bioseguridad & Número & $\%$ \\
$\begin{array}{l}\text { Utilizan zapatos adecuados (no sandalias) en } \\
\text { sus clases prácticas en laboratorios, clínicas, } \\
\text { hospitales, etc. }\end{array}$ & 1016 & 96 \\
$\begin{array}{l}\text { Limpian y desinfectan el área de trabajo antes } \\
\text { de comenzar su actividad }\end{array}$ & 759 & 72 \\
$\begin{array}{l}\text { Realizan cambio de gabacha diariamente } \\
\begin{array}{l}\text { Respetan la prohibición de consumir alimentos } \\
\text { o agua en las prácticas de laboratorio, clínicas, } \\
\text { hospitalarias, etc. }\end{array}\end{array}$ & 388 & 37 \\
$\begin{array}{l}\text { Depositan el material bioinfeccioso en bolsas } \\
\text { rojas para ser eliminado }\end{array}$ & 390 & 37 \\
\hline
\end{tabular}

Gráfico 10. Porcentaje de estudiantes por carrera que han adquirido conocimientos sobre bioseguridad

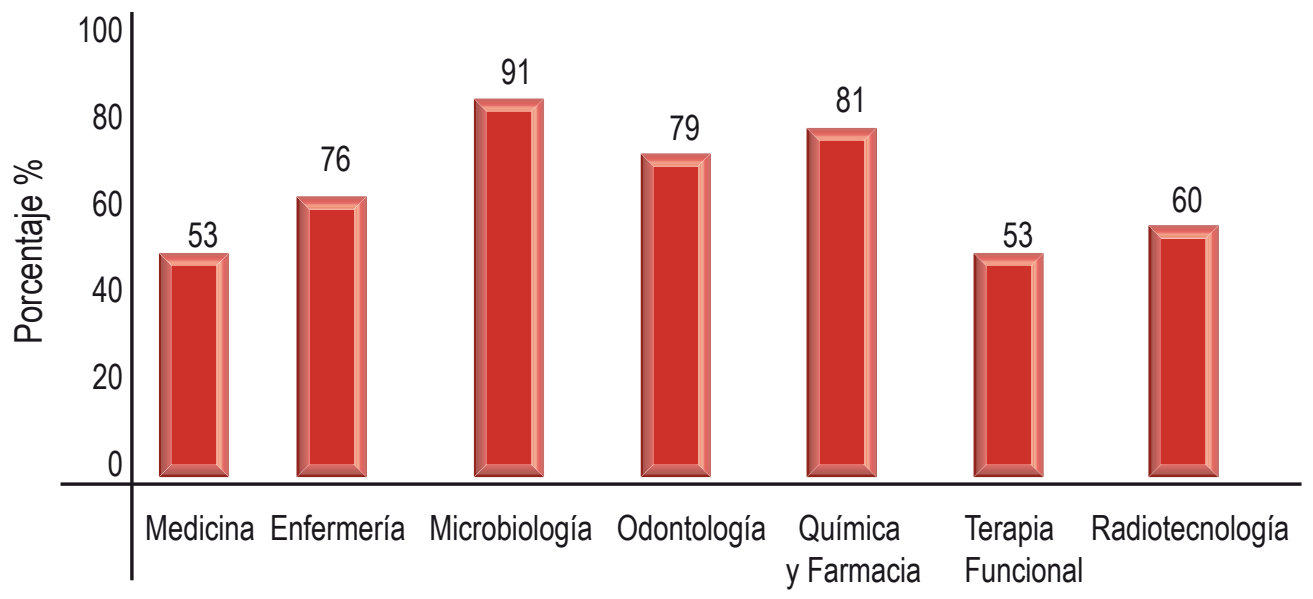




\section{DISCUSIÓN}

Se define bioseguridad como el conjunto de principios, normas, técnicas y prácticas que deben aplicarse para la protección del individuo, la comunidad y el medioambiente, para protegerse ante el contacto natural 0 accidental con agentes que son potencialmente nocivos. También es considerada como la aplicación de conocimientos, técnicas y equipamientos para prevenir a personas, laboratorios, áreas hospitalarias y medioambiente de la exposición a agentes potencialmente infecciosos o considerados de riesgo biológico (OMS, 2005).

Los estudiantes de las carreras del área de la salud están expuestos a riesgos biológicos: transmisión de infecciones por contacto directo con los microorganismos provenientes de cultivos, uso frecuente de objetos punzocortantes, muestras biológicas, sangre y otros fluidos corporales, desechos bioinfecciosos, exposición a aerosoles y salpicaduras, contacto con fómites (materiales, superficies, equipo de protección personal contaminado), derrames biológicos, desechos biológicos. Riesgo químico: debido a la exposición frecuente a sustancias químicas tóxicas, irritantes, explosivas o cancerígenas utilizadas en las prácticas de laboratorio o clínicas; desinfectantes, exposición a sustancias químicas para el revelado de radiografías, medicamentos citostáticos, derrames químicos, desechos químicos. Riesgo físico: radiaciones ionizantes, mecheros de gas, sobrecarga de las líneas eléctricas, uso de electrodos, calor, etc.

En consecuencia, el $83 \%$ de los estudiantes identifica algún tipo de riesgo biológico. En todas las carreras se observó la exposición al riesgo químico, sin embargo, solamente el $5 \%$ señala este riesgo, de igual manera el $6.8 \%$ de los estudiantes que participaron en la investigación pertenecen a la Carrera de Radiotecnología; pero solamente el $4 \%$ de los estudiantes de esta Carrera manifestó estar expuesto a las radiaciones ionizantes.

La capacidad de reconocer los peligros es el primer paso para prevenir la exposición ocupacional y ambiental, igualmente, desarrollar competencias asociadas con el uso de barreras primarias y secundarias para prevenir la exposición a materiales peligrosos, así como las competencias relativas a la descontaminación y gestión de residuos peligrosos, precauciones universales, precauciones con las muestras de sangre, manipulación de ropa contaminada, manejo de agujas o instrumentos punzocortantes, manejo de derrames biológicos y químicos, exposición a radiaciones ionizantes, manejo de la hidroterapia, desinfección, esterilización, etc. (Rivera, 2005). 
La hepatitis $B$, la hepatitis $C$, el sida y la tuberculosis se encuentran entre los riesgos más significativos para los trabajadores de la salud en general (OPS, 2005). El $70 \%$ de los estudiantes identifica el virus de la inmunodeficiencia humana $(\mathrm{VIH})$ y el $43 \%$ el virus de la hepatitis como transmitidos por fluidos corporales; aunque se observa un mayor conocimiento sobre el $\mathrm{VIH}$, probablemente porque en el ámbito académico y en la sociedad en general se habla más de esta enfermedad que de la hepatitis $B$.

Los aerosoles y salpicaduras son los medios más eficaces de transmisión de enfermedades infecciosas debido a su pequeño tamaño y a que se producen en múltiples procedimientos; al respecto, menos del $50 \%$ de los estudiantes conoce las medidas de bioseguridad para protegerse de los aerosoles y salpicaduras, situación que aumenta el riesgo, ya que no están siendo entrenados en procedimientos críticos como la centrifugación, el trasvasado de sustancias, el uso de instrumentos dentro de la boca de los pacientes, etc.

El $58 \%$ identifica medidas adecuadas en caso de sufrir una pinchadura con un objeto cortopunzante potencialmente infeccioso, porcentaje muy bajo considerando que la administración de salud y bienestar ocupacional (OSHA) determina cinco actividades principales relacionadas con las heridas con objetos punzocortante: el manejo de agujas, la administración de inyectables, la extracción de sangre, el reencapuchamiento de agujas y el manejo de desperdicios; siendo las agujas las implicadas con mayor frecuencia en las heridas con objeto punzocortante (CDC, 2011). Los estudiantes realizan diariamente estos procedimientos, sin embargo, solo el $58 \%$ identifica medidas de protección.

La mayoría de los estudiantes identifican la gabacha, guantes y, en menor grado, el uso de la mascarilla como la forma de protegerse de la transmisión de enfermedades transmitidas por fluidos y líquidos corporales. El $61 \%$ reporta el uso de EPP durante sus prácticas de laboratorio 0 en las clínicas, porcentaje que es muy bajo considerando que este uso es la barrera principal de protección contra los riesgos bioinfecciosos, químicos y algunos físicos.

Conocen muy poco sobre los desinfectantes utilizados en la práctica y sobre las hojas de seguridad de las sustancias químicas utilizadas en sus carreras; exceptuando la Carrera de Química y Farmacia, en la cual un 84 \% reporta conocerlas. Más del $90 \%$ está de acuerdo con la afirmación: "El personal del área de la salud está expuesto a más riesgos laborales que otro tipo de profesionales" y el $84 \%$ se considera en riesgo de adquirir una infección durante el proceso enseñanza-aprendizaje, lo que demuestra una percepción de riesgo alta. La importancia que debemos ofrecerle 
a este aspecto es que quien no percibe el riesgo, no asume una posición constructiva de enfrentamiento; los individuos que se sienten seguros y aquellos cuyas actitudes reflejan algún grado de conocimiento sobre el riesgo, experimentan menos obstáculos para modificar su ambiente que los que responden con actitudes de defensa.

Las razones por las que los estudiantes se consideran en riesgo de contaminarse 0 de sufrir un daño durante el proceso enseñanza- aprendizaje son las siguientes: puede ocurrir un accidente (70 \%), hay compañeros descuidados (54\%) y porque hay muchos estudiantes en poco espacio (53 \%). Se observa que los estudiantes tienden a culpabilizar a otros del riesgo al que están expuestos y no consideran sus actitudes como un factor que predispone a la exposición al riesgo.

En consecuencia, es indispensable que los profesionales de la salud en formación conozcan y pongan en práctica las precauciones universales como el uso de guantes, lavado de manos, uso de gabacha, delantales y anteojos protectores, etc. Estas son las medidas higiénicas generales que tienen como objetivo evitar la transmisión de enfermedades a través de los fluidos corporales (Hernández, 2006), ya que solamente el $47 \%$ utiliza siempre las medidas de bioseguridad, siendo los estudiantes de las Carreras de Odontología y Microbiología los que más las usan, lo que está en relación directa con el entrenamiento que han recibido sobre bioseguridad.

No se encontró relación directa entre percepción de riesgo y la aplicación de medidas de bioseguridad, pero sí se halló relación entre haber recibido en la carrera conocimientos adecuados de bioseguridad y la aplicación de medidas de bioseguridad en el proceso enseñanza- aprendizaje ( $p$ 0.0001).

Las razones por las cuales los estudiantes aplican las medidas de bioseguridad son principalmente el deseo de protegerse ante los riesgos existentes y porque han adquirido los conocimientos necesarios para llevar a cabo prácticas de protección. El grado de protección que experimentan los estudiantes en sus respectivas carreras es bajo, siendo los estudiantes de la Carrera de Microbiología los que se sienten más protegidos.

El material bioinfeccioso o contaminado que sale de laboratorios, clínicas, hospitales, clínicas odontológicas, etc. debe ser depositado y esterilizado antes de salir del lugar en donde se generó, para ser eliminado, realizar una correcta clasificación de los residuos para su adecuado manejo en las bolsas correspondientes y desechar agujas u otros objetos punzocortantes sin reencapuchar en recipientes cerrados, rígidos, 
rotulados y no perforables; pues de otro modo se tendrían problemas serios en la salud de los trabajadores asistenciales (Soto, 2004). Solamente el $47 \%$ de los estudiantes deposita los desechos bioinfecciosos en los recipientes adecuados, lo cual pone en riesgo al personal de limpieza de las diferentes carreras y a las personas que recogen los desechos reciclables en el depósito municipal.

Se considera que entre las causas más frecuentes de infección en el personal de salud se encuentran: accidentes de trabajo al manipular las muestras, negligencia e inobservancia de reglamentos al manipular agentes infecciosos, no disponer de medios adecuados de protección, personal inadecuadamente entrenado, etc. Se detectó que entre los casos de accidentes laborales por fluidos biológicos, un $54 \%$ fueron de gravedad moderada, de los cuales el $57 \%$ fue producto de un objeto punzocortante como las agujas hipodérmicas (Mohamad, 2003).

En el presente estudio se observó que el $11 \%$ (120) de los estudiantes ha sufrido alguna pinchadura con objeto punzocortante durante sus actividades académicas llevadas a cabo en laboratorios de la UNAH o en clínicas y hospitales, el $55 \%$ reportó el incidente al profesor, sin embargo, el $45 \%$ no notificó el incidente sufrido, por lo que se presume que no se tomaron medidas de contención para prevenir algún efecto adverso ante la exposición biológica con objeto punzocortante contaminado.

La bioseguridad es una acción educativa y como tal puede ser representada por un sistema enseñanza-aprendizaje. En ese sentido, podemos entenderla como un proceso de adquisición de contenidos y habilidades con el objetivo de preservar la salud del hombre y del medioambiente (Ferreira, Barrozo y García, 2004). Es importante lograr la concienciación adecuada del personal que trabaja en áreas de alto riesgo del hospital sobre la importancia del cumplimiento de las normas de bioseguridad, pues de otro modo se tendrían problemas serios en la salud de los trabajadores asistenciales (Soto, 2004).

Una encuesta de conocimiento realizada en el país por expertos internacionales en mayo de 2010 (Sánchez, 2010), durante el Primer Taller Nacional de Bioseguridad organizado por la Escuela de Microbiología, muestra que el $43 \%$ de los asistentes no habían participado en cursos o talleres de entrenamiento en bioseguridad en el último año; además, el $56 \%$ de la audiencia manifestó sentirse en riesgo de adquirir una infección durante su quehacer profesional (Sánchez, 2011). Más del 50 \% reporta haber recibido capacitación en bioseguridad, siendo los estudiantes de las Carreras de Microbiología, Química y Farmacia y Odontología los que han recibido mayor número de cursos o talleres de bioseguridad. 


\section{CONCLUSIONES}

1. El conocimiento sobre los riesgos y las medidas de prevención que tienen los estudiantes del área de la salud se enfoca principalmente al riesgo biológico, lo mismo se observa en el conocimiento que tienen sobre las medidas de prevención. Existe poca información sobre el riesgo químico y físico al que se exponen diariamente durante el proceso enseñanza aprendizaje. En general, se observa aplicación adecuada de las medidas de bioseguridad.

2. Existe una adecuada percepción del riesgo de los estudiantes de las carreras del área de la salud, el $89 \%$ considera que el personal de salud está más expuesto a riesgos laborales que otro tipo de carreras. Sin embargo, esta percepción no les lleva necesariamente a tomar medidas de prevención, pues no se encontró relación directa entre percepción de riesgo y la práctica de medidas de protección.

3. En términos generales, los estudiantes perciben un nivel de protección bajo en sus carreras y otros, lo que es aún más preocupante, desconocen el nivel de protección con que trabajan.

4. No se encontró relación directa entre la percepción de riesgo y las prácticas de prevención que realizan los estudiantes del área de la salud de la UNAH. De acuerdo a la hipótesis planteada se esperaría que los estudiantes, al tener una mayor percepción de riesgo, su comportamiento relacionado con la prevención se manifestara en cumplimiento de las medidas de prevención. La discusión al respecto lleva a plantear que la capacidad del estudiante para tomar decisiones que lo conduzcan a adoptar medidas de prevención está sujeta a múltiples variables: el conocimiento, existencia de normas y materiales, equipo, super-visión, etc. Sí se encontró relación directa entre haber adquirido conocimientos adecuados de bioseguridad en las carreras y la aplicación de medidas de bioseguridad. 


\section{RECOMENDACIONES}

1. Cada carrera debe identificar y capacitar a los estudiantes acerca de los riesgos biológicos, físicos y químicos a los que se exponen los estudiantes en el proceso enseñanza-aprendizaje y las medidas de contención para disminuir el riesgo; además, elaborar los procedimientos operativos estándares de las diferentes técnicas de prevención, incluirlas en un manual de bioseguridad y dar entrenamiento a los estudiantes sobre el uso y manejo de los mismos.

2. Mejorar la percepción de riesgo de los estudiantes aumentando el conocimiento de los riesgos a los que se exponen, fomentando la cultura de bioseguridad entre docentes, estudiantes y personal administrativo. También realizar evaluaciones de riesgo, monitorear el cumplimiento de las normas, asignar presupuesto para insumos y materiales de bioseguridad para que las prácticas de bioseguridad se implementen en cada una de las carreras del área de la salud.

3. Incorporar la bioseguridad como un eje transversal en cada una de las carreras del área de la salud, iniciar la capacitación y el entrenamiento en las diferentes prácticas de prevención desde los primeros períodos académicos de los estudiantes; asimismo, en cada asignatura entrenar, monitorear y exigir el cumplimiento de las medidas de bioseguridad.

4. Gestionar para que sea posible contar con los controles de ingeniería, administrativos y del material y equipo necesario para llevar a cabo procesos que conduzcan a la bioseguridad personal y colectiva, así como la del medioambiente, integrar un comité de bioseguridad, elaborar un reglamento interno de bioseguridad y nombrar un comité de vigilancia para que vele por el cumplimiento de las prácticas de bioseguridad.

5. Modificar o replantear los contenidos de bioseguridad en las diferentes asignaturas y cursos a fin de que el alumno sea capaz de identificar y categorizar los riesgos biológicos, químicos o físicos a los que se enfrenta durante su formación académica y su vida profesional. Además, el alumno deberá comprender y seleccionar los diferentes tipos de barreras para el control o mitigación de tales riesgos y proponer soluciones integrales y adecuadas para el manejo integral del riesgo. 


\section{REFERENCIAS}

CDC. (2011). Guidelines for Biosafety Laboratory Competency and the Association of Public Health Laboratories. Morbidity and Mortality Weekly Report, 60(15), 1-11. Ferreira, M.; Barrozo, M. y García, L. (2004). Educación en bioseguridad en Brasil: reflexiones y competencias necesarias. Revista Cubana Salud Pública, 30, 3.

Hernández, V. (2006). Intervención educativa para incrementar los conocimientos sobre bioseguridad en el personal de enfermería de una institución hospitalaria. Revista Cubana Enfermería, 22(2), 62-71.

Organización Mundial de la Salud. (2005). Manual de bioseguridad en el laboratorio. Ginebra.

Organización Panamericana de la Salud. (2005). Salud y seguridad de los trabajadores del sector salud: manual para gerentes y administradores. Washington, D.C. Rivera Valdivia, Á.; Paz, M.; Bratti, L. y Chinchilla, A. (Abril de 2005). Accidentes ocupacionales y conocimiento sobre precauciones universales en internos universitarios costarricences. Acta Médica Costarricense, 47(2), 89-93. San José.

Sánchez, A.; Zelaya, A.; Enríquez, L. y Bautista, R. (2010). Informe técnico de entrenamiento y evaluación de bioseguridad. Canadá: Brock University.

Sánchez, A.; Kaufman, S.; Zelaya, A.; Enríquez, L. y Canales, M. (2011). Biosafety Competencies in Developing Countries: The Role of Universities. Applied Biosafety, 16(4), 240-252. EE.UU.

Soto, V. y Olano, E. (2004). Conocimiento y cumplimiento de medidas de bioseguridad en personal de enfermería. Chiclayo: Hospital Nacional Almanzor Aguinaga. 